ISSN: 0210-1696

DOI: http://dx.doi.org/10.14201/scero20164745772

\title{
CONSTRUCCIÓN Y VALIDACIÓN DE LA ESCALA DE SATISFACCIÓN LABORAL (ESL) EN CENTROS ESPECIALES DE EMPLEO
}

\section{Construction and Validation of Employee Job Satisfaction Scale in Special Needs Employment Centres}

José Francisco Lukas Mujika

Universidad del País Vasco. Facultad de Educación, Filosofía y Antropología.

Departamento de Métodos de Investigación y Diagnóstico en Educación. Avenida de Tolosa, 70. 20018 San Sebastián

jf.lukas@ehu.eus

Juan EtxeberRia Murgiondo

Universidad del País Vasco. Facultad de Educación, Filosofía y Antropología.

Departamento de Métodos de Investigación y Diagnóstico en Educación

Xabier Galarreta Lasa

Universidad del País Vasco. Facultad de Educación, Filosofía y Antropología.

Departamento de Didáctica y Organización Escolar

Luis LiZASOAIN HeRnÁNDEZ

Universidad del País Vasco. Facultad de Educación, Filosofía y Antropología.

Departamento de Métodos de Investigación y Diagnóstico en Educación

Esta investigación ha sido financiada por la empresa GUREAK.

Recepción: 21 de junio de 2016

Fecha de aceptación definitiva: 12 de enero de 2017

Resumen: Es tarea de los Centros Especiales de Empleo (CEE) crear oportunidades laborales que permitan a sus trabajadores construir las mejores condiciones de calidad de 
vida, tanto en el contexto del propio puesto de trabajo como en su vida en general. Ambos aspectos han de ser tomados en consideración a la hora de medir el impacto de las oportunidades que aporta el puesto de trabajo. En este contexto se hace imprescindible contar con instrumentos validados para evaluar, entre otros, la satisfacción de los trabajadores con respecto a la aportación que significa trabajar en un CEE. En este marco, se han analizado la pertinencia y el valor de una herramienta que ha sido construida con la intención de valorar la satisfacción con las condiciones laborales de los trabajadores de los CEE de Gureak. La Escala de Satisfacción Laboral (ESL) elaborada ha sido aplicada a una muestra de 563 personas con discapacidad intelectual (PCDI) y personas con enfermedad mental (PCEM) incluidas en el Régimen Especial de Empleo (REE). Para la validación de la escala se ha analizado el funcionamiento de los ítems y la fiabilidad y validez. En todos los casos, se han obtenido índices adecuados. De la misma manera, se ha realizado el estudio de la estructura factorial de la escala, utilizando tanto el análisis de componentes principales (ACP) como el análisis factorial confirmatorio (AFC), obteniéndose cuatro factores en la escala: Bienestar en el trabajo, Condiciones laborales, Disponibilidad de Información y Apoyo y Autodeterminación.

Palabras Clave: satisfacción labral; validación; Centro Especial de Empleo; discapacidad intelectual; enfermedad mental.

Aвstract: It is the function of Special Needs Employment Centres (CEE in its Spanish acronym) to create job opportunities that enable workers to construct the best conditions for quality of life, both within the context of the job itself as well as within that of their lives in general. Both aspects have to be taken into account when measuring the impact of the opportunities afforded by the job. In this context it is essential to have at hand validated instruments in order to evaluate, amongst other factors, the satisfaction of employees regarding the contribution made by working in a CEE. Within this framework, analysis was made of the usefulness and value of a tool which was constructed with the intention of evaluating the level of satisfaction with the working conditions of the employees in the Gureak CEE. The Employee Job Satisfaction Scale (ESL in its Spanish acronym) that was drawn up was applied to a sample of 563 persons with intellectual disability (PCDI in its Spanish acronym) and persons suffering from mental illness (PCEM in its Spanish acronym) and falling under the remit of the Special Needs Employment Directive (REE in its Spanish acronym). For the validation of the scale the functioning of items of reliability and validity were analysed. In each case, suitable indices were obtained. Likewise, research on the factorial structure of the scale was undertaken, using both principal components analysis (PCA) and confirmatory factorial analysis (CFA), obtaining thus four factors in the scale: Wellbeing at Work, Labor Conditions, Information Availability, and Support and Self-Determination.

KEYWORDS: employee job satisfaction; validation; Special Needs Employment Centre; intellectual disability; mental illness. 


\section{Introducción}

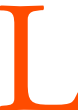

os Centros Especiales de Empleo (CEE) tratan de hacer realidad las orientaciones del artículo 35 de la Ley General de los Derechos de las Personas con Discapacidad y de su inclusión social (2013): "Las personas con discapacidad tienen derecho al trabajo, en condiciones que garanticen la aplicación de los principios de igualdad de trato y no discriminación”. Para ello construyen propuestas que permitan el desarrollo de "una actividad productiva de bienes o de servicios, participando regularmente en las operaciones del mercado, y tienen como finalidad el asegurar un empleo remunerado para las personas con discapacidad” (art. 43).

El grupo empresarial Gureak (www.grupogureak.com) genera y gestiona oportunidades laborales adaptadas a las personas con discapacidad, prioritariamente para personas con discapacidad intelectual en Gipuzkoa. La organización viene aportando oportunidades laborales a personas en situación de discapacidad desde 1972 (actualmente participan 4.600 personas con Discapacidad Intelectual o Enfermedad Mental). Desde 2006 Gureak está realizando un importante esfuerzo en el desarrollo de prácticas orientadas desde la Planificación Centrada en la Persona (PCP) a través del desarrollo y seguimiento de Planes Personales de Apoyo para sus trabajadores (Heredia, 2016).

Es tarea de los CEE crear oportunidades laborales que permitan a sus trabajadores construir las mejores condiciones de calidad de vida, tanto en el contexto del propio puesto de trabajo como en su vida en general. Ambos aspectos han de ser tomados en consideración a la hora de medir el impacto de las oportunidades que aporta el puesto de trabajo. En este contexto se hace imprescindible contar con instrumentos validados para evaluar, entre otros, la satisfacción de los trabajadores con respecto a la aportación que significa trabajar en un CEE. En este marco, se han analizado la pertinencia y el valor de una herramienta que ha sido construida con la intención de valorar la satisfacción con las condiciones laborales de los trabajadores de los CEE de Gureak.

Es un objetivo en la vida de todas las personas lograr las mayores cotas de satisfacción en los diferentes ámbitos de la vida. La posibilidad y/o la oportunidad de participar en procesos laborales, ya sea desde un empleo o en una ocupación, se convierten en uno de los principales referentes a la hora de valorar nuestra satisfacción. En el ámbito de las políticas, los servicios y las intervenciones que se relacionan con las situaciones de discapacidad, la Calidad de Vida se ha convertido en un referente significativo a la hora de orientar las prácticas y sus valoraciones.

Desde las propuestas de Schalock y Verdugo $(2003,2007)$ existe un consenso en relación a las dimensiones que definen el constructo de la Calidad de Vida. Estas dimensiones se convierten en elementos referenciales para la planificación y evaluación de las prácticas, también laborales, relacionadas con la discapacidad. Por ello, la calidad de vida es un indicador fundamental de los servicios que prestan oportunidades a las personas en situación de discapacidad. El concepto tiene un importante impacto en las organizaciones y de manera contundente en los resultados personales (Schalock y Verdugo, 2007).

(C) Ediciones Universidad de Salamanca / CC BY-NC-ND

Siglo Cero, vol. 47 (4), n. ${ }^{\circ}$ 260, 2016, octubre-diciembre, pp. 57-72 
En los últimos años encontramos estudios que evalúan y valoran dicha calidad de vida, tanto desde una perspectiva objetiva como subjetiva (Verdugo, 2006; Beyer, 2010). Rollins, Bond, Jones, Kukla y Collins (2011) estudian la importancia de las redes sociales establecidas en el centro de trabajo entre los empleados con enfermedades mentales severas. Pagán (2014), en una línea similar, revisa la importancia de las cuestiones no pecuniarias en relación a la satisfacción laboral. Pérez, Alcover y Chambel (2015) estudian las actitudes hacia el trabajo de las personas con discapacidades, resaltando la importancia de unir el apoyo familiar al que presta la propia organización. Por su parte, Baumgärtner, Dwertmann, Boehm y Bruch (2015) se centran en el rol de la flexibilidad estructural percibida a la hora de analizar la satisfacción laboral de este colectivo.

Algunos de estos procesos de valoración han tenido como participantes a personas adultas en situación de discapacidad que trabajaban en servicios de empleo ordinario o de trabajo protegido, ya sea éste en régimen de empleo especial o mediante servicios ocupacionales.

En este contexto, se considera el concepto Satisfacción como una variante o concreción de los elementos o dimensiones generales de la Calidad de Vida, aplicados o concretados en alguno de los ámbitos laborales señalados con anterioridad. Según Sirgy et al. (2001) la calidad de la vida laboral se deriva de experimentar satisfacción en cuatro dominios: la satisfacción en el entorno laboral (condiciones saludables, salario, etc.), los requisitos de trabajo (tareas requeridas, conflictos, autonomía, trabajo en equipo, etc.), la supervisión (retroalimentación, apoyo, etc.) y programas (programas de mejora de la calidad de vida laboral, la flexibilidad de los horarios) (Flores, Jenaro, Orgaz y Martín, 2011). De la misma manera, Flores, Jenaro, González-Gil y GarcíaCalvo (2010) señalan que los términos asociados a la calidad de vida laboral son las características del trabajo, la satisfacción laboral, el estrés ocupacional, las características organizativas, el clima organizacional y las condiciones de trabajo.

Como podrá comprobarse, la Escala de Satisfacción Laboral construida por Gureak en realidad recoge los aspectos señalados por los autores citados previamente, por lo que se considera que la intención era medir la satisfacción, como concreción de la calidad de vida laboral.

La incorporación de iniciativas de desarrollo de la Calidad de Vida y de la Satisfacción Laboral por parte de organizaciones que desarrollan los CEE es una buena referencia, tanto para la propia orientación de los centros, como para poder encontrar indicadores para su transformación, en la búsqueda de adecuar sus ofertas al mejor interés de las personas participantes. Siendo éste un camino complejo (Tamarit, 2015), no deja de ser imprescindible para abordar el reto de acompañar al proceso de construcción de ciudadanos con plenos derechos.

\section{Antecedentes de la Escala de Satisfacción Laboral (ESL)}

En los Talleres Protegidos Gureak la herramienta que orienta el desarrollo de las prácticas PCP adopta el formato de Planes Personales de Apoyo (PPA). Estos Planes

(C) Ediciones Universidad de Salamanca / CC BY-NC-ND

Siglo Cero, vol. 47 (4), n. ${ }^{\circ}$ 260, 2016, octubre-diciembre, pp. 57-72

$$
-60-
$$


están construidos a partir de las 8 dimensiones de Calidad de Vida (Schalock y Verdugo, 2003). En los PPA, Gureak realizó una operativización de estas dimensiones en 8 categorías fácilmente identificables en la práctica, tanto para los profesionales como para los trabajadores, que además incorporaban el Bienestar Emocional y la Autodeterminación de manera transversal. Estas categorías se utilizaron como referencia a lo largo del proceso, tanto para la valoración inicial de la satisfacción de los operarios, como para la construcción posterior del PPA.

A continuación presentamos las 8 categorías definidas y el número de ítems que incorporaba la versión de 2006 del cuestionario de satisfacción laboral:

ACTIVIDAD LABORAL: Situación en la que se encuentra el operario y las expectativas que tiene respecto a las actividades que desarrolla en el taller, cuáles son sus necesidades en el puesto de trabajo, en la tarea que realiza, etc. (7 ítems).

CONDICIONES LABORALES: Opiniones y expectativas en relación a cuestiones como el horario, las vacaciones, el salario, la estabilidad en el trabajo, la información, las oportunidades de promoción, etc. (6 ítems).

FORMACIÓN: Hace referencia expresa a la formación que recibe el operario, a la utilidad de lo que aprende y del trabajo que desarrolla, a las oportunidades de cambio de actividad, etc. (4 ítems).

SALUD: Ámbito relacionado con la percepción del bienestar físico que experimenta la persona, las condiciones y la seguridad del puesto de trabajo percibido por el operario, etc. (6 ítems).

Relaciones: Ámbito referido a las relaciones interpersonales, al tipo de relación establecida con los encargados y compañeros, a la calidad de las mismas, etc. (7 ítems).

Acceso, sAlida y vestuarios: Cuestiones referidas a la forma de acceder al taller, su opinión sobre los vestuarios, al momento de la entrada y la salida, etc. (6 ítems).

COMEDOR Y DESCANSOs: Aspectos relacionados con el servicio de comedor, de los tiempos de descanso, del tiempo libre, etc. (5 ítems).

COMUNIDAD: Ámbitos relacionados con aspectos sociales y con otras dimensiones no laborales que influyen en las expectativas y necesidades de los operarios. (2 ítems).

Este cuestionario de satisfacción es el que los trabajadores cumplimentaban como inicio del proceso y fijación de sus necesidades y deseos, a partir de los cuales comenzaba la elaboración de su Plan Personal. A su vez, el cuestionario ha servido de soporte para la construcción de la herramienta actual de la Escala de Satisfacción Laboral (ESL). El concepto subyacente de satisfacción laboral adoptado ha derivado en que, de las 8 dimensiones iniciales, se hayan seleccionado los ítems vinculados a las 4 primeras: Actividad de Taller, Condiciones Laborales, Formación y Salud. Y en las preguntas que se formulan, se puede detectar un tratamiento más explícito de la dimensión de autodeterminación que anteriormente era abordada de manera transversal. 
Partiendo del PPA se ha realizado una selección de las dimensiones y de los ítems, quedando configurado el instrumento de la siguiente manera:

\begin{tabular}{|c|c|}
\hline Dimensiones & Ítems \\
\hline Actividad taller & $\begin{array}{l}\text { 1. ¿Te gustan las tareas que realizas? } \\
\text { 10. ¿Hay buenas relaciones y buen ambiente en el trabajo? } \\
\text { 12. ¿Se tiene en cuenta tu opinión en el puesto de trabajo? } \\
\text { 13. ¿Puedes tomar decisiones y participar en el trabajo? }\end{array}$ \\
\hline Condiciones laborales & $\begin{array}{l}\text { 4. ¿Tu sueldo es conforme a las tareas que realizas? } \\
\text { 5. ¿Estás satisfecho con el horario? } \\
\text { 6. ¿Estás satisfecho con el calendario? } \\
\text { 8. ¿Cuando estás trabajando te encuentras bien contigo mismo? } \\
\text { 14. ¿Tienes información suficiente del puesto de trabajo? } \\
\text { 15. ¿Si tienes algún problema puedes comunicarlo para resolverlo? }\end{array}$ \\
\hline Formación & $\begin{array}{l}\text { 2. ¿Aprendes cosas nuevas que luego te sirven en el trabajo? } \\
\text { 3. ¿El equipo de taller te facilita o ayuda a que avances o progre- } \\
\text { ses en tu trabajo? } \\
\text { 11. ¿Tu encargado te apoya cuando lo necesitas? }\end{array}$ \\
\hline Salud & $\begin{array}{l}\text { 7. ¿Estás seguro en el trabajo? } \\
\text { 9. ¿El taller te ayuda a estar tranquilo? }\end{array}$ \\
\hline Ítem criterio & 16. ¿Cómo estás de satisfecho en general con el trabajo? \\
\hline
\end{tabular}

A estos 15 ítems se les añadió el ítem 16 para ser utilizado como ítem criterio para el cálculo de las diferentes propiedades estadísticas de la escala.

El objetivo del presente estudio fue diseñar, construir y validar la Escala de Satisfacción Laboral (ESL). Para la consecución del mismo, se estudió el funcionamiento de los ítems, la fiabilidad y validez de la escala, así como su estructura interna.

\section{Método}

\subsection{Participantes}

La población objeto de este estudio han sido los trabajadores del grupo Gureak. Más en concreto, son personas con discapacidad intelectual (PCDI) y personas con enfermedad mental (PCEM) incluidas en el Régimen Especial de Empleo (REE).

Las empresas del grupo que han participado en este estudio son 9. La población en abril de 2015 se componía de 1.015 sujetos distribuidos en las 9 empresas, tal y como figura en la Tabla 1.

La selección de los integrantes de la muestra se realizó de forma aleatoria en los talleres protegidos Gureak, mientras que en el resto de empresas el estudio fue censal. El número final de unidades fue de 563 personas, de las que 220 eran mujeres $(39,1 \%)$ y 343 hombres $(60,9 \%)$, con edades comprendidas entre los 20 y los 65 años.

(C) Ediciones Universidad de Salamanca / CC BY-NC-ND

Siglo Cero, vol. 47 (4), n. ${ }^{\circ}$ 260, 2016, octubre-diciembre, pp. 57-72

$$
-62-
$$




\begin{tabular}{|l|r|r|}
\hline \multicolumn{1}{|c|}{ Tabla 1. Población y muestra de participantes en el estudio } \\
\hline \multicolumn{1}{|c|}{ Empresa } & \multicolumn{1}{|c|}{ Ree } & Muestra \\
\hline Talleres Protegidos Gureak, S. A. & 645 & 233 \\
\hline Gupost, S. A. U. Publicidad directa & 30 & 30 \\
\hline Goiplastik, S. L. U. & 9 & 9 \\
\hline Guregas Carburantes, S. L. U. & 29 & 29 \\
\hline Gureak Oiartzun, S. L. U. & 35 & 29 \\
\hline Goroldi, S. L. U. & 64 & 59 \\
\hline Galant Garbitasuna, S. L. U. & 156 & 130 \\
\hline Gureserbi, S. L. U. & 44 & 42 \\
\hline Gureak Ostalaritza, S. L. U. & 3 & 2 \\
\hline Total & 1015 & 563 \\
\hline
\end{tabular}

444 personas $(78,9 \%)$ con discapacidad intelectual y $119(21,1 \%)$ con alguna enfermedad mental. La media del grado de discapacidad es de $45,98 \%$.

\subsection{Diseño}

Para la validación de la escala se ha seguido la Teoría Clásica de los Tests. Para ello, por una parte, se ha analizado el funcionamiento estadístico de los ítems, es decir, su discriminación, homogeneidad y validez. Por otra, se ha analizado el funcionamiento de la escala en su conjunto, es decir, su coeficiente de fiabilidad, su validez y su estructura factorial.

\subsection{Procedimiento}

La escala ha sido administrada por una profesional con varios años de experiencia como proveedora de apoyos en Gureak y con un amplio conocimiento en la relación con personas con discapacidad.

La decisión de que la encuesta fuera administrada por medio de una única entrevistadora se tomó con el fin de garantizar la comprensión de los ítems y el formato de respuesta. Para ello se preparó un material complementario que consta de ejemplos y anexos (que ayudan a entender los enunciados) y material visual de apoyo para el formato de respuesta. Antes de iniciar la sesión, la entrevistadora realizaba una actividad que aseguraba que el formato de respuesta era comprendido por la persona.

Una vez la entrevistadora se aseguraba que la persona entendía los diferentes ítems y comprendía el formato de respuesta, se iniciaba la recogida de datos. Antes de comenzar con la entrevista, se le explicaba a la persona que sus respuestas serían totalmente confidenciales y que intentara, por lo tanto, contestar con la mayor sinceridad posible. 
Figura 2. Formato de la hoja de respuestas

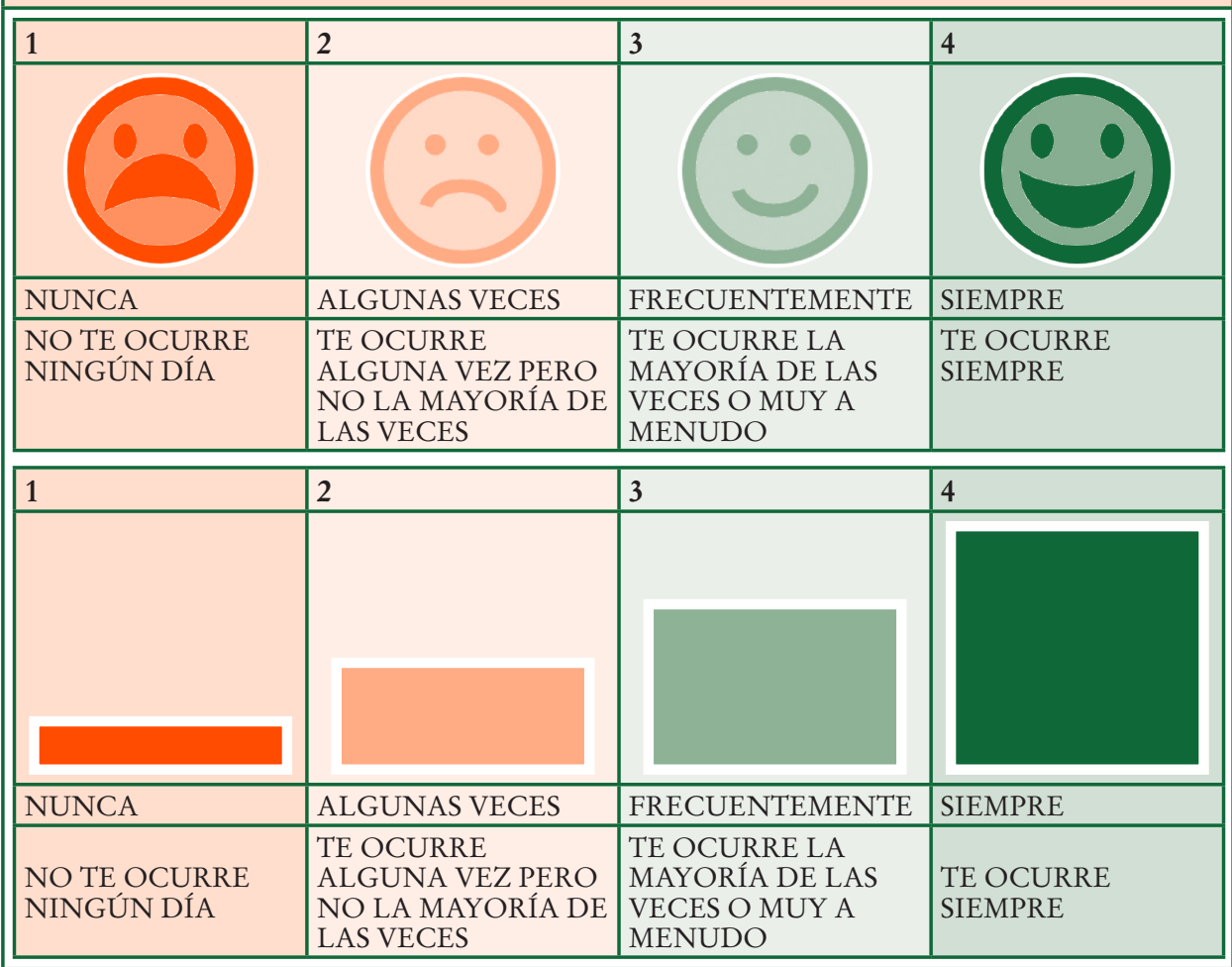

Estos procedimientos de aplicación asistida de un instrumento son empleados con cierta frecuencia a la hora de interactuar con participantes de estas características. Por ejemplo, Acosta, Vales, Echeverria, Serrano y García (2013) aplican un procedimiento muy similar con apoyos gráficos y visuales a la hora de estudiar la fiabilidad y validez del Cuestionario de Calidad de Vida (WHOQOL-OLD). Finlay y Antaki (2012) analizan los mecanismos de interacción del personal investigador con participantes adultos con discapacidades intelectuales. Desde una perspectiva algo distinta, Zhang, McCrickard y Tanis (2012) elaboran una aplicación para teléfonos móviles con objeto de facilitar la aplicación de cuestionarios a personas con discapacidades cognitivas en la que emplean abundantes recursos gráficos.

\subsection{Análisis estadísticos}

Una vez efectuada la recogida de datos, se procedió a la informatización de los mismos. Tras la depuración inicial de los datos, se realizaron los análisis estadísticos, para

(C) Ediciones Universidad de Salamanca / CC BY-NC-ND

Siglo Cero, vol. 47 (4), n. ${ }^{\circ}$ 260, 2016, octubre-diciembre, pp. 57-72

$$
-64-
$$


lo que se utilizó la versión 23 del paquete estadístico SPSS y el complemento AMOS. Las respuestas dadas a cada uno de los ítems del cuestionario representan una escala ordinal, por lo que para los diferentes análisis se emplearon coeficientes de correlación policóricos y para el análisis factorial exploratorio (AFE) el complemento R-Factor para este tipo de variables (Basto y Pereira, 2012). Dada la similitud de los resultados obtenidos mediante estas técnicas con los de las habituales para escala de intervalos, en aras de la interpretabilidad de los resultados presentamos los mismos considerando los valores de las variables como indicadores de una escala de intervalos.

Para la validación de la escala, se han realizado los siguientes análisis:

- Para obtener el índice de homogeneidad de los ítems, se ha calculado la correlación de Pearson entre cada ítem y la puntuación total en la escala.

- Para obtener el índice de validez de los ítems, se ha calculado la correlación de Pearson entre cada ítem y el ítem criterio (el ítem 16).

- Para obtener la fiabilidad de la escala, se ha calculado el coeficiente de fiabilidad, utilizándose para ello el coeficiente de consistencia interna o Alfa de Cronbach.

- Para obtener la validez de la escala, se han calculado las correlaciones policóricas y de Pearson entre las puntuaciones totales de la misma y la puntuación obtenida en el ítem criterio.

- Para el estudio de la estructura factorial de la escala, se ha realizado un análisis de componentes principales (ACP) que se ha complementado con el análisis factorial confirmatorio (AFC) realizado mediante Modelos de Ecuaciones Estructurales usando AMOS.

\section{Resultados}

El análisis de la homogeneidad de los ítems hace referencia a la consistencia de cada uno de ellos con respecto al cuestionario. Para su cálculo se ha correlacionado cada uno de los ítems con las puntuaciones totales obtenidas en la prueba (ver Tabla 2).

\begin{tabular}{|l|c|c|}
\hline \multicolumn{3}{|c|}{ Tabla 2. Índices de homogeneidad (IH) y de validez (IV) de los ítems } \\
\hline \multicolumn{2}{|c|}{ (TH } & $I V$ \\
\hline 1. ¿Te gustan las tareas que realizas? &, 492 &, 550 \\
\hline 2. ¿Aprendes cosas nuevas que luego te sirven en el trabajo? &, 428 &, 376 \\
\hline $\begin{array}{l}\text { 3. ¿El equipo del taller te facilita o ayuda a que avances o progreses en tu } \\
\text { trabajo? }\end{array}$ &, 500 &, 354 \\
\hline 4. ¿Tu sueldo es conforme a las tareas que realizas? &, 328 &, 263 \\
\hline 5. ¿Estás satisfecho/a con el horario ? &, 363 &, 334 \\
\hline 6. ¿Estás satisfecho/a con el calendario? &, 373 &, 328 \\
\hline 7. ¿Estás seguro/a en el trabajo? &, 265 &, 171 \\
\hline 8. ¿Cuando estás trabajando te encuentras bien contigo mismo/a? &, 531 &, 546 \\
\hline 9. ¿El taller (brigada/centro de trabajo) te ayuda a estar tranquilo/a? &, 547 &, 522 \\
\hline
\end{tabular}

(C) Ediciones Universidad de Salamanca / CC BY-NC-ND

Siglo Cero, vol. 47 (4), n. ${ }^{\circ}$ 260, 2016, octubre-diciembre, pp. 57-72

$$
-65-
$$


TABLA 2. Índices de homogeneidad (IH) y de validez (IV) de los ítems

\begin{tabular}{|l|c|c|}
\hline & $I H$ & $I V$ \\
\hline 10. ¿Hay buenas relaciones y un buen ambiente en el trabajo? &, 467 &, 350 \\
\hline \begin{tabular}{l|c} 
11. ¿Tu encargado/a te apoya cuando lo necesitas? \\
12. ¿Se tiene en cuenta tu opinión en el puesto de trabajo?
\end{tabular} &, 515 &, 384 \\
\hline $\begin{array}{l}\text { 13. ¿Puedes tomar decisiones y participar en el trabajo? } \\
\text { 14. ¿Tienes información suficiente del puesto del trabajo (taller/brigada/ } \\
\text { empresa)? }\end{array}$ &, 168 &, 353 \\
\hline \begin{tabular}{l} 
15. ¿Si tienes algún problema puedes comunicarlo para resolverlo? \\
\hline
\end{tabular} &, 470 &, 305 \\
\hline
\end{tabular}

Como puede comprobarse, la gran mayoría de los ítems se correlacionan positivamente con la puntuación total de la prueba y, en general, puede decirse que los valores obtenidos están dentro de lo que se considera como aceptable. No obstante, los ítems 7 (“¿Estás seguro/a en el trabajo?”) y 13 (“¿Puedes tomar decisiones y participar en el trabajo?”) son los que obtienen un índice de consistencia o de homogeneidad más bajo. Su valor se sitúa en un caso en 0,168 (ítem 13) y en 0,265 el 7.

Con respecto al índice de validez de los ítems, se ha calculado la correlación de cada uno de ellos con el ítem criterio (ítem 16) para comprobar si realmente miden lo que pretenden medir. En la Tabla 2 pueden observarse los valores obtenidos.

Los índices de validez son positivos y estadísticamente significativos. Ello nos lleva a concluir que los ítems han funcionado correctamente. No obstante, una vez más, los ítems 7 y 13 aparecen con una correlación que no llega a 0,2.

Se ve, por tanto, que, en lo que concierne a la homogeneidad y validez, estos dos ítems muestran un comportamiento débil. El ítem 7 se centra en cuestiones de seguridad en el trabajo y el otro en la posibilidad de toma de decisiones. Son dos cuestiones muy específicas y, de hecho, como se verá más adelante, el ítem 13 (que aquí es el que obtiene los valores más bajos) en el análisis de la estructura factorial muestra un comportamiento específico y claramente diferenciado del resto. Esto puede estar señalando la necesidad de que los procesos de toma de decisiones por parte de este colectivo de trabajadores sean estudiados con mayor profundidad.

La fiabilidad de una prueba hace referencia a la consistencia de las puntuaciones que ofrece y a su exactitud y precisión (Lukas, 1998). Su cálculo viene determinado por el Coeficiente de Fiabilidad, que puede ser hallado utilizando diversos procedimientos. En el caso que nos ocupa, se ha calculado el Coeficiente de Consistencia Interna (Alfa de Cronbach), cuyo resultado es de 0,802, que para este tipo de pruebas debe considerarse más que aceptable.

La validez de una escala hace referencia al análisis de hasta qué punto la misma mide aquello que pretende. Para garantizar la misma se han seguido dos procedimientos.

Por un lado, en la construcción de la escala y en su posterior validación tanto interna como externa se ha garantizado por personas expertas que el contenido recoge aquello que pretende medir.

Por otro lado, para la validación empírica se añadió un ítem criterio 16 (“¿Cómo estás de satisfecho/a en general con el trabajo?”) que engloba lo que pretende medir la

(C) Ediciones Universidad de Salamanca / CC BY-NC-ND

Siglo Cero, vol. 47 (4), n. ${ }^{\circ}$ 260, 2016, octubre-diciembre, pp. 57-72

$$
-66-
$$


suma del resto de los ítems. De esta manera, se ha podido correlacionar la puntuación global obtenida en la escala con la puntuación obtenida en el ítem criterio obteniendo un valor de $0,659(p=, 000)$, por lo que se puede concluir que el resultado obtenido permite acreditar la validez de la escala. Es cierto que el haber preguntado por este ítem criterio en el mismo momento de aplicación que el resto del cuestionario puede conllevar un efecto de incremento de la varianza compartida. Pero dadas las limitaciones inherentes al diseño, de cara a la aplicación del instrumento a los participantes, no fue posible realizarlo de otra manera. En cualquier caso, este posible efecto debe ser tenido en cuenta a la hora de valorar los resultados de validez.

El estudio de la estructura dimensional de la prueba se ha realizado en primer lugar mediante AFE, empleando el módulo R-Factor para ACP de variables ordinales. Como antes ha sido expuesto, la solución factorial así obtenida es muy similar a la resultante del ACP habitual, por lo que aquí se presentan los resultados de este último una vez realizada la rotación Promax.

Las Tablas 3 y 4 muestran y definen la estructura factorial de los 15 ítems. Como puede observarse, el primer componente estaría compuesto por 6 ítems $(1,2,7,8,9$ y 10). Tras efectuar una rotación Promax pues es lógico suponer que los componentes estén correlacionados, las cargas factoriales de los ítems asignados a este primer componente son claras excepto en el caso del ítem 2 (0,354). Pero, dada la formulación del

\begin{tabular}{|l|c|c|c|c|}
\hline \multirow{2}{*}{} & \multicolumn{5}{c|}{ TABLA 3. Análisis de componentes principales } \\
\cline { 2 - 5 } & 1 & 2 & 3 & 4 \\
\hline ítem1 &, 782 &,- 178 &, 092 &, 052 \\
\hline ítem2 &, 354 &, 048 &, 196 &, 238 \\
\hline ítem3 &, 103 &, 265 &, 383 &, 206 \\
\hline ítem4 &,- 162 &, 049 &, 843 &,- 120 \\
\hline ítem5 &, 231 &,- 107 &, 540 &,- 038 \\
\hline ítem6 &, 224 &, 073 &, 506 &,- 434 \\
\hline ítem7 &, 542 &, 167 &,- 270 &,- 304 \\
\hline ítem8 &, 784 &,- 126 &, 064 &, 140 \\
\hline ítem9 &, 660 &, 174 &,- 103 &, 100 \\
\hline ítem10 &, 491 &, 128 &, 093 &,- 019 \\
\hline ítem11 &, 020 &, 667 &, 083 &, 100 \\
\hline ítem12 &,- 048 &, 638 &,- 020 &, 366 \\
\hline ítem13 &, 151 &,- 003 &,- 161 &, 804 \\
\hline ítem14 &, 059 &, 716 &,- 165 &,- 241 \\
\hline ítem15 &,- 100 &, 753 &, 125 &,- 067 \\
\hline $\begin{array}{l}\text { Método de extracción: Análisis de componentes principales. } \\
\text { Método de rotación: Normalización Promax con Kaiser. }\end{array}$ & \\
\hline
\end{tabular}

(C) Ediciones Universidad de Salamanca / CC BY-NC-ND

Siglo Cero, vol. 47 (4), n. ${ }^{\circ}$ 260, 2016, octubre-diciembre, pp. 57-72

$$
-67-
$$


mismo, y que es en este factor donde se da la mayor carga, se ha optado por incluirlo en este primer componente.

El segundo está compuesto por 4 ítems (11, 12, 14 y 15). En este caso las cargas factoriales son elevadas y se presentan sólo en este componente excepto en el ítem 12, que también presenta una carga no desdeñable en el cuarto componente. Por su formulación podría haber acompañado al ítem 13 en el cuarto componente relativo a la autodeterminación. Pero la magnitud de la carga (que luego se verá confirmada en el AFC, y siguiendo las mejoras de ajuste sugeridas en modification fit del propio análisis) impele a asignarlo a esta segunda dimensión.

El factor 3 está a su vez compuesto por otros 4 ítems (3, 4, 5 y 6). Los cuatro muestran una clara adscripción al factor a pesar de que la carga del ítem 3 es relativamente baja en comparación con los otros 3 ítems.

Por último, esta estructura factorial se completa con un cuarto componente compuesto en el que carga casi exclusivamente el ítem 13. Como antes se ha señalado, se trata de un ítem importante que aborda la cuestión de la toma de decisiones por parte de las personas. Pero su comportamiento estadístico es de gran dispersión y claramente diferenciado del resto.

En la Tabla 4 se muestran también los valores del Alfa de Cronbach para los 3 componentes compuestos por más de un ítem, pues lógicamente para el cuarto factor no tiene sentido su cálculo. Dado el bajo número de ítems que componen cada factor, los valores del alfa no son tampoco muy elevados, lo que se acentúa en el segundo componente con un valor de sólo 0,554. Ello puede ser debido a que en el mismo se ha incluido el ítem 3, cuyo comportamiento es menos sólido que el del resto.

Tabla 4. Definición y coeficiente de fiabilidad de 3 de los 4 factores

FACTOR 1: Bienestar en el trabajo. Fiabilidad: 0,712.

Ítem 1. ¿Te gustan las tareas que realizas?

Ítem 2. ¿Aprendes cosas nuevas que luego te sirven en el trabajo?

Ítem 7. ¿Estás seguro/a en el trabajo?

Ítem 8. ¿Cuando estás trabajando te encuentras bien contigo mismo/a?

Ítem 9. ¿El taller (brigada/centro de trabajo) te ayuda a estar tranquilo/a?

Ítem 10. ¿Hay buenas relaciones y un buen ambiente en el trabajo?

FACTOR 2: Condiciones laborales. Fiabilidad: 0,554.

Ítem 3. ¿El equipo del taller te facilita o ayuda a que avances o progreses en tu trabajo?

Ítem 4. ¿Tu sueldo es conforme a las tareas que realizas?

Ítem 5. ¿Estás satisfecho/a con el horario?

Ítem 6. ¿Estás satisfecho/a con el calendario?

FACTOR 3: Disponibilidad de Información y apoyo. Fiabilidad: 0,658.

Ítem 11. ¿Tu encargado/a te apoya cuando lo necesitas?

Ítem 12. ¿Se tiene en cuenta tu opinión en el puesto de trabajo?

Ítem 14. ¿Tienes información suficiente del puesto del trabajo (taller/brigada/empresa)?

Ítem 15. ¿Si tienes algún problema puedes comunicarlo para resolverlo?

FACTOR 4: Autodeterminación.

Ítem 13. ¿Puedes tomar decisiones y participar en el trabajo? 
El análisis exploratorio se complementa con el Análisis Factorial Confirmatorio. A continuación (Figura 3) se muestra el modelo de mejor ajuste. Se ha trabajado con tres factores (hemos eliminado del análisis el ítem 13). Los índices de ajuste, que podemos considerar satisfactorios, fueron $\mathrm{X}^{2}=124,71$ con 68 grados de libertad, con un valor de $\mathrm{X}^{2} / \mathrm{gl}=1,834$, mientras que el valor de $\mathrm{CFI}=0,962$, GFI $=0,921, \mathrm{NFI}=0,970$ y un valor de RMSEA $=0,039$, indicadores todos ellos de un buen ajuste (Schreider et al., 2006; Hooper et al., 2008; Kaplan, 2009; Kline, 2011). Así mismo se puede apreciar una buena relación entre los tres factores.

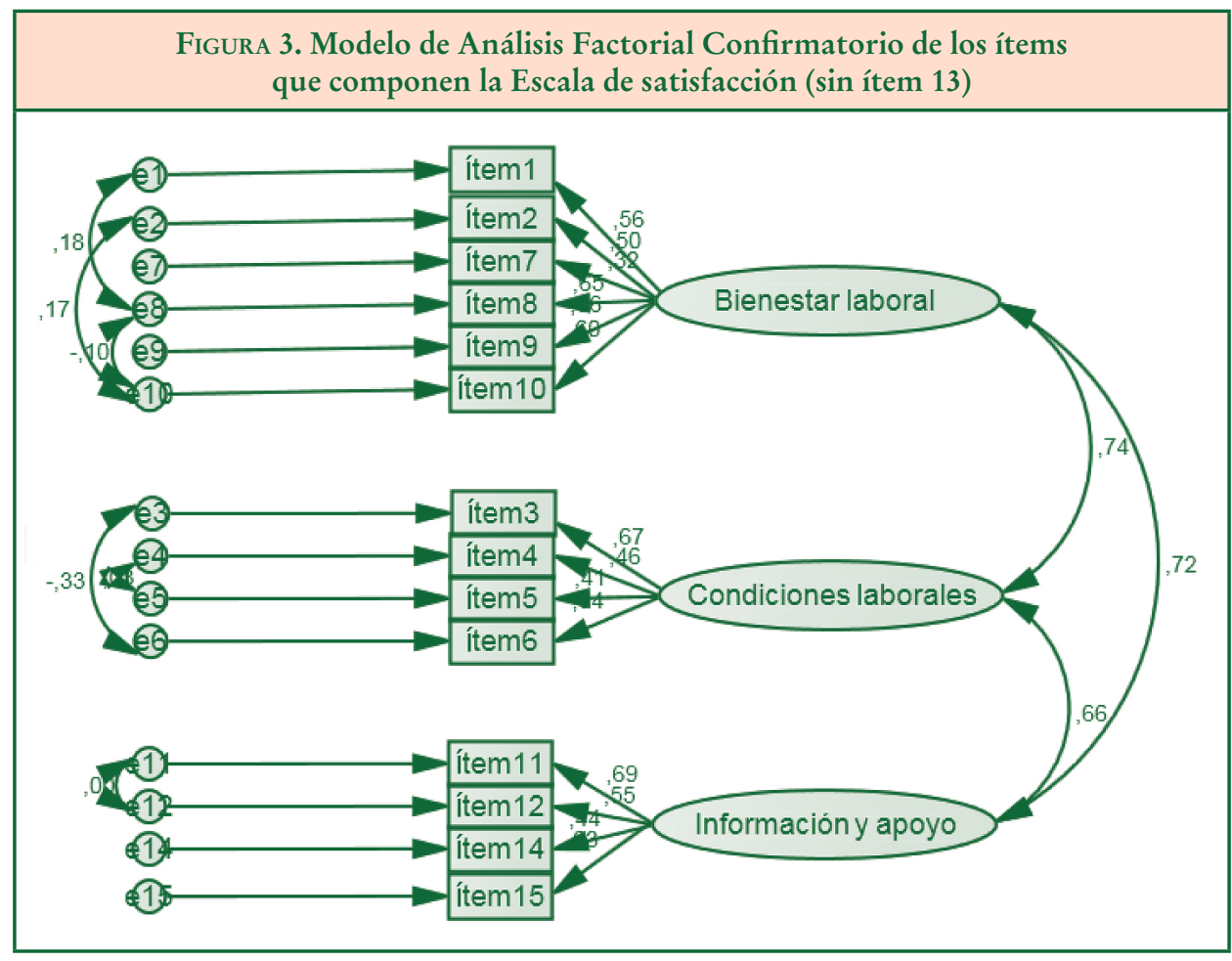

\section{Discusión y conclusiones}

Los participantes que han respondido a los cuestionarios conforman una muestra representativa de las empresas para las que se pretende validar el cuestionario. La muestra está compuesta por la práctica totalidad de personas que trabajan en las empresas de menos trabajadores, y por el $40,77 \%$ del total en el caso de los Talleres Protegidos Gureak (263 de un total de 645). Han participado en el estudio 563 sobre un total de 1.015, lo que representa el 55,46\%. Este hecho nos posibilita obtener conclusiones válidas para el colectivo objeto de estudio. 
Con respecto al funcionamiento de la escala, se ha comprobado que la gran mayoría de los ítems que conforman la misma tienen un buen comportamiento estadístico, es decir, poseen un grado de discriminación suficiente, son homogéneos y válidos con respecto al ítem criterio utilizado. De la misma manera, la precisión y estabilidad de las puntuaciones está garantizada dado que los coeficientes de fiabilidad obtenidos así lo demuestran. La validez de la escala se ha asegurado tanto por el procedimiento de construcción que se ha seguido como por la relación de las puntuaciones obtenidas con el ítem criterio. En cualquier caso, al respecto, no hay que olvidar que la aplicación simultánea de los 15 ítems y del ítem criterio puede llevar aparejado un efecto de incremento de la varianza compartida.

La definición teórica de la que se partió (versión 2006) establecía una estructura de 43 ítems agrupados en 8 dimensiones. Por razones de aplicabilidad y de eficiencia se optó por elaborar un instrumento más reducido asumiendo las limitaciones y dificultades estadísticas (fiabilidad, estructura dimensional) que tal decisión podría acarrear.

$\mathrm{El}$ análisis estadístico de las respuestas obtenidas muestra que el cuestionario adopta una estructura de 4 factores. El primer factor, denominado Bienestar en el Trabajo, se puede entender como satisfacción con las tareas realizadas y con el clima de seguridad y de relaciones del taller. El segundo factor, Condiciones Laborales, recoge aquellos elementos en torno al salario, horarios y calendario junto con las oportunidades de formación y desarrollo. El tercer factor, Disponibilidad de Información y Apoyo, identifica aquellos elementos relacionados con la proximidad de los recursos de apoyo y conocimiento de la organización. Por último, el cuarto factor, Autodeterminación (integrado únicamente por el ítem 13), hace referencia a la toma de decisiones.

$\mathrm{El}$ instrumento aborda múltiples aspectos relativos a la satisfacción laboral que en algunos casos no están interrelacionados: seguridad, niveles salariales, autodeterminación, etc. Por razones de índole práctica se optó por elaborar un instrumento breve que cubriese esta diversidad de aspectos. Ello trae como consecuencia que cada factor está compuesto por un número muy limitado de ítems, siendo el cuarto factor el caso extremo. Con la finalidad de seguir estudiando las diversas dimensiones subyacentes, convendría ampliar en lo posible el número de ítems siendo conscientes de que ello puede conllevar problemas y dificultades de aplicación.

Como conclusión, se puede afirmar que la Escala de Satisfacción Laboral (ESL) es un instrumento fiable y válido, susceptible de ser utilizado como herramienta en Centros de Empleo Especial. Por medio del mismo, pueden obtenerse evidencias que permitan optimizar las ofertas de los centros de empleo a la vez que faciliten la construcción de proyectos personales para sus trabajadores. Obviamente, las posibilidades diagnósticas de esta herramienta deberían ser complementadas con estudios adicionales y con información recogida utilizando procedimientos cualitativos.

\section{Referencias bibliográficas}

Acosta, C. O., Vales, J. J., Echeverría, S. B., Serrano, D. M. y García, R. (2013). Confiabilidad y validez del Cuestionario de Calidad de Vida (WHOQOL-OLD) en adultos mayores mexicanos. Psicología y Salud, 23 (2), 241-250.

() Ediciones Universidad de Salamanca / CC BY-NC-ND

Siglo Cero, vol. 47 (4), n. ${ }^{\circ} 260,2016$, octubre-diciembre, pp. 57-72 
Basto, M. y Pereira, J. M. (2012). An SPSS R-Menu for Ordinal Factor Analysis. Journal of Statistical Software, 46 (4). doi: 10.18637/jss.v046.i04.

Baumgärtner, M. K., Dwertmann, D. G., Boehm, S. A. y Bruch, H. (2015). Job satisfaction of employees with disabilities: The role of perceived structural flexibility. Human Resource Management, 54 (2), 323-343. doi: 10.1002/hrm.21673.

Beyer, S., Brown, T., Akandi, R. y Rapley, M. A. (2010). Comparison of Quality of Life Outcomes for People with Intellectual Disabilities in Supported Employment, Day Services and Employment Enterprises. Journal of Applied Research in Intellectual Disabilities, 23, 290-295.

Finlay, W. M. L. y ANTAKi, C. (2012). How staff pursue questions to adults with intellectual disabilities. Journal of Intellectual Disability Research: JIDR, 56 (4), 361-370. https://doi. org/10.1111/j.1365-2788.2011.01478.x.

Flores, N., Jenaro, C., González-Gil, F. y García-Calvo, P. M. (2010). Análisis de la calidad de vida laboral en trabajadores con discapacidad. Zerbitzuan, 47, 95-10.

Flores, N., Jenaro, C., Orgaz, M. B. y Martín, M. V. (2011). Understanding Quality of Working Life of Workers with Intellectual Disabilities. Journal of Applied Research in Intellectual Disabilities, 24, 133-141.

Franco, T., Martínez, J. y Pisonero, S. (2006). Satisfacción de las personas en Lantegi Batuak. Siglo Cero, 218, 79-92.

GuREAK (2006). Documento marco: Definición y mejora del proceso de individualización en la división industrial de TP Gureak. Documento interno.

Heredia, T. (2016). Aportaciones del modelo de Calidad de Vida en los procesos de individualización en los servicios de empleo y ocupación. Análisis del caso Gureak. Tesis doctoral inédita. UPV-EHU.

Hooper, D., Coughlan, J. y Mullen, M. R. (2008). Structural Equation Modelling: Guidelines for Determining Model Fit. The Electronic Journal of Business Research Methods, 6 (1), 53-60.

Kaplan, D. (2009). Structural Equation Modeling (2 ${ }^{\text {nd }}$ ed.): Foundations and Extensions. Thousand Oaks, USA: SAGE Publications.

Kline, R. (2011). Principles and practice of structural equation modeling (3 ${ }^{\text {rd }}$ ed.). New York, London: The Guilford Press.

Lukas, J. F. (1998). Análisis de items y de tests con ITEMAN. Lejona: Servicio de Publicaciones de la Universidad del País Vasco.

PAGÁN, R. (2014). What makes workers with disabilities happy? The importance of non-pecuniary characteristics. Health Economics, 23 (2), 241-247. doi: 10.1002/hec.2905.

Pérez, V., Alcover, C. y Chambel, M. J. (2015). Job attitudes among workers with disabilities: The importance of family support in addition to organizational support. Work: Journal of Prevention, Assessment E Rehabilitation, 51 (4), 817-826. doi: 10.3233/WOR-141905.

Real Decreto Legislativo 1/2013, de 29 de noviembre, por el que se aprueba el Texto Refundido de la Ley General de derechos de las personas con discapacidad y de su inclusión social. $B O E$ 3 de diciembre de 2013.

Rollins, A. L., Bond, G. R., Jones, A. M., Kukla, M. y Collins, L. A. (2011). Workplace social networks and their relationship with job outcomes and other employment characteristics for people with severe mental illness. Journal of Vocational Rehabilitation, 35 (3), 243-252.

Schalock, R. L. y Verdugo, M. Á. (2003). Calidad de vida. Manual para profesionales de la educación, salud y servicios sociales. Madrid: Alianza editorial. 
Schalock, R. L. y Verdugo, M. Á. (2007). El concepto de Calidad de vida en los servicios y apoyos para personas con discapacidad intelectual. Siglo Cero, 224, 21-37.

Schreider, J. B., Stage, F. K., King, J., Nora, A. y Barlow, E. A. (2006). Reporting structural equation modeling and confirmatory factor analysis results: a review. The Journal of Education Research, 99 (6), 323-337.

Sirgy, M. J., Efraty, D., Siegel, P. et al. (2001). A New Measure of Quality of Work Life (QWL) Based on Need Satisfaction and Spillover Theories. Social Indicators Research, 55, 241. doi: 10.1023/A:1010986923468.

TAMARIT, J. (2015). La transformación de los servicios hacia la calidad de vida. Una iniciativa de innovación social de Feaps. Siglo Cero, 255, 47-71.

Verdugo, M. Á., Jordán de Urriés, F. B., Jenaro, C., Caballo C. y Crespo, M. (2006). Quality of Life of Workers with an Intellectual Disability in Supported Employment. Journal of Applied Research in Intellectual Disabilities, 19, 309-316.

Zhang, G., McCrickard, D. S. y Tanis, S. (2012). Designing a Mobile Survey Application for People with Cognitive Disabilities. Recuperado a partir de http://citeseerx.ist. psu.edu/viewdoc/citations;jsessionid=D548BFE9ABDD9F0E9D84D899764B15BF?d oi=10.1.1.310.9343. 